\title{
DYNAMICS OF THE ALPHA CEN SYSTEM.
}

J. ANOSOVA ${ }^{1}$, V. ORLOV ${ }^{2}$

1. National Astronomical Observatory, Tokyo 181, Japan;

2. Astronomical Institute, St. Petersburg University,

Bibliotechnaya pl. 2, 198904 St. Petersburg, Peterhof, Russia

The triple star system $\alpha$ Cen $A B$ and Proxima Cen - the component $C$ - is the nearest to the Sun. The study of its dynamics has shown that this system is probable non-chance. The motion of the component $C$ (Proxima) with respect to the centre of mass of the pair $A B$ is hyperbolic with the probability $P=1.0$. We observe, therefore, a slow passage of $C$ close to the pair $A B$. We propose the hypothesis that this system is a part of the stellar moving group. We list the probable members of this group amongst the nearby stars. Amongst them we have the binaries Gliese 140.1 and 676, the triple system ADS 10288 (Gliese 649.1), and the six single stars. The probability to find by chance these stars inside the velocity space cube with the side of $20 \mathrm{~km} / \mathrm{s}$ around $\alpha C e n$ is equal to about $2 \%$. 